\title{
Measuring (1,3)- $\beta$-D-glucan in tracheal aspirate, bronchoalveolar lavage fluid, and serum for detection of suspected Candida pneumonia in immunocompromised and critically ill patients: a prospective observational study
}

Kang-Cheng Su ${ }^{1,2,3}$, Kun-Ta Chou ${ }^{1,2}$, Yi-Han Hsiao ${ }^{1,3}$, Ching-Min Tseng ${ }^{3,4}$, Vincent Yi-Fong Su', Yu-Chin Lee ${ }^{5}$, Diahn-Warng Perng ${ }^{1,6^{*}+}$ and Yu Ru Kou ${ }^{3 *+}$

\begin{abstract}
Background: While Candida pneumonia is life-threatening, biomarker measurements to early detect suspected Candida pneumonia are lacking. This study compared the diagnostic values of measuring levels of $(1,3)-\beta-D-$ glucan in endotracheal aspirate, bronchoalveolar lavage fluid, and serum to detect suspected Candida pneumonia in immunocompromised and critically ill patients.

Methods: This prospective, observational study enrolled immunocompromised, critically ill, and ventilated patients with suspected fungal pneumonia in mixed intensive care units from November 2010 to October 2011. Patients with D-glucan confounding factors or other fungal infection were excluded. Endotracheal aspirate, bronchoalveolar lavage fluid and serum were collected from each patient to perform a fungal smear, culture, and D-glucan assay.

Results: After screening 166 patients, 31 patients completed the study and were categorized into non-Candida pneumonia/non-candidemia ( $n=18)$, suspected Candida pneumonia $(n=9)$, and non-Candida pneumonia/candidemia groups $(n=4)$. D-glucan levels in endotracheal aspirate or bronchoalveolar lavage were highest in suspected Candida pneumonia, while the serum D-glucan level was highest in non-Candida pneumonia/candidemia. In all patients, the D-glucan value in endotracheal aspirate was positively correlated with that in bronchoalveolar lavage fluid. For the detection of suspected Candida pneumonia, the predictive performance (sensitivity/specificity/D-glucan cutoff [pg/m/]) of D-glucan in endotracheal aspirate and bronchoalveolar lavage fluid was 67\%/82\%/120 and 89\%/86\%/130, respectively, accounting for areas under the receiver operating characteristic curve of 0.833 and 0.939 (both $P<0.05$ ), respectively. Measuring serum D-glucan was of no diagnostic value (area under curve $=0.510, P=0.931$ ) for the detection of suspected Candida pneumonia in the absence of concurrent candidemia.

(Continued on next page)
\end{abstract}

\footnotetext{
* Correspondence: dwperng@vghtpe.gov.tw; yrkou@ym.edu.tw

${ }^{\dagger}$ Equal contributors

'Department of Chest Medicine, Taipei Veterans General Hospital, No.201, Sec. 2, Shipai Rd., Beitou Dist., Taipei City 11217, Taiwan, Republic of China ${ }^{3}$ Institute of Physiology, School of Medicine, National Yang-Ming University, No.155, Sec.2, Linong St., Beitou Dist., Taipei City 11221, Taiwan, Republic of China

Full list of author information is available at the end of the article
} 
(Continued from previous page)

Conclusions: D-glucan levels in both endotracheal aspirate and bronchoalveolar lavage, but not in serum, provide good diagnostic values to detect suspected Candida pneumonia and to serve as potential biomarkers for early detection in this patient population.

Keywords: (1,3)- $\beta$-D-glucan, Bronchoalveolar lavage, Candida pneumonia, Critically ill patients, Diagnosis, Intensive care unit

\section{Background}

Candida pneumonia (CP) is life-threatening and has been associated with a high attributable mortality [1]. However, the definitive diagnosis is rarely established before overwhelming sepsis or death. Conventionally, a lung biopsy has been proposed to confirm CP [2, 3], but the procedure is too risky for patients in intensive care units (ICUs) because of the high prevalence of thrombocytopenia and coagulopathy [4]. Growth of respiratory Candida spp. in immunocompromised, cancer-afflicted, and critically ill patients is frequently found, but it is usually considered colonization rather than $\mathrm{CP}$ [5]. Indeed, growth of respiratory Candida spp. lacks specificity to diagnose CP [6-9], and adds little value to optimizing $\mathrm{CP}$ management $[10,11]$. Recently, growing evidence has challenged the dogma that respiratory Candida spp. acts as a bystander in ICU patients [12]. The presence of respiratory Candida spp. has been shown to be associated with increased bacterial pneumonia development [13-16], selection of multidrug-resistant bacteria [17], and worse ICU outcomes [14, 18-20]. Accordingly, measurements of biomarkers with diagnostic value for the early detection of suspected CP are urgently needed.

Measurements of serum $(1,3)-\beta$-D-glucan (BDG), the common fungal wall antigen, can now be accomplished within hours. Measuring serum BDG has emerged as a rapid assessment to facilitate the diagnosis of invasive fungal infection, and it has proven to provide indirect mycological evidence for invasive fungal infection by a task force consensus [3]. A recent meta-analysis of 2979 patients included from 16 studies showed that using serum BDG levels to diagnose invasive fungal infection could attain a pooled sensitivity and specificity of 76.8 and $85.3 \%$, respectively, and an area under the receiver operating characteristic curve (AUROC) of 0.89 [20]. However, none of these studies focused on respiratory Candida spp. infection. Additionally, it is conceivable that measuring BDG levels in respiratory specimens might be more accurate and specific for the diagnosis of pulmonary infection compared to measuring BDG levels in serum. Thus, the diagnostic value of measuring BDG levels in serum or respiratory specimens to detect suspected CP remains unclear.

In this study, we conducted a prospective, observational study aiming to compare the diagnostic value of measuring BDG levels in endotracheal aspirate (TA), bronchoalveolar lavage (BAL) fluid, and serum for the detection of suspected CP in immunocompromised and critically ill patients.

\section{Methods \\ Study design}

This prospective, observational study was conducted at medical and surgical ICUs in a medical center, Taipei Veterans General Hospital, in Taiwan, from November 2010 to October 2011. Eligible patients were recruited, and specimens of TA, BAL (see Additional file 1 for detail methods), and serum were collected from each individual patient on the enrollment day. BAL with or without transbronchial lung biopsy (TBLB) was a routine procedure at the discretion of the treating physicians in patients with unrecognized pulmonary lesions in our ICU. All the specimens were submitted to a bacterial culture, a fungal smear and culture, and a BDG assay. Fungal stain was applied routinely to inspect fungal structures microscopically. This study was approved by the institutional review board of Taipei Veterans General Hospital (IRB approval ID: 201011002IA) and signed informed consents were obtained from all participants.

\section{Patients}

Adult, mechanically ventilated ICU patients with suspected fungal pneumonia were recruited if they met all of the following conditions: presence of appropriate host factors defined by the task force consensus (see Additional file 1 for details about host factors) [3], non-responding or progressive pulmonary lesions despite management with broad-spectrum antibiotics for 48 to $72 \mathrm{~h}$, and signature on an inform consent form. Patients were excluded if they withdrew consent, lacked respiratory specimens, had established diagnoses other than $\mathrm{CP}$, or presented with factors that might confound the BDG assay (Fig. 1; see Additional file 1 for details about confounding factors) [10, 21-23]. The application of antifungal treatment depended on the treating team, which was blinded to the BDG results until ICU discharge. The final diagnosis was determined by at least 2 different ICU intensivists independently, including the primary physicians. A conference was held to determine the final diagnosis if a discrepancy existed. 


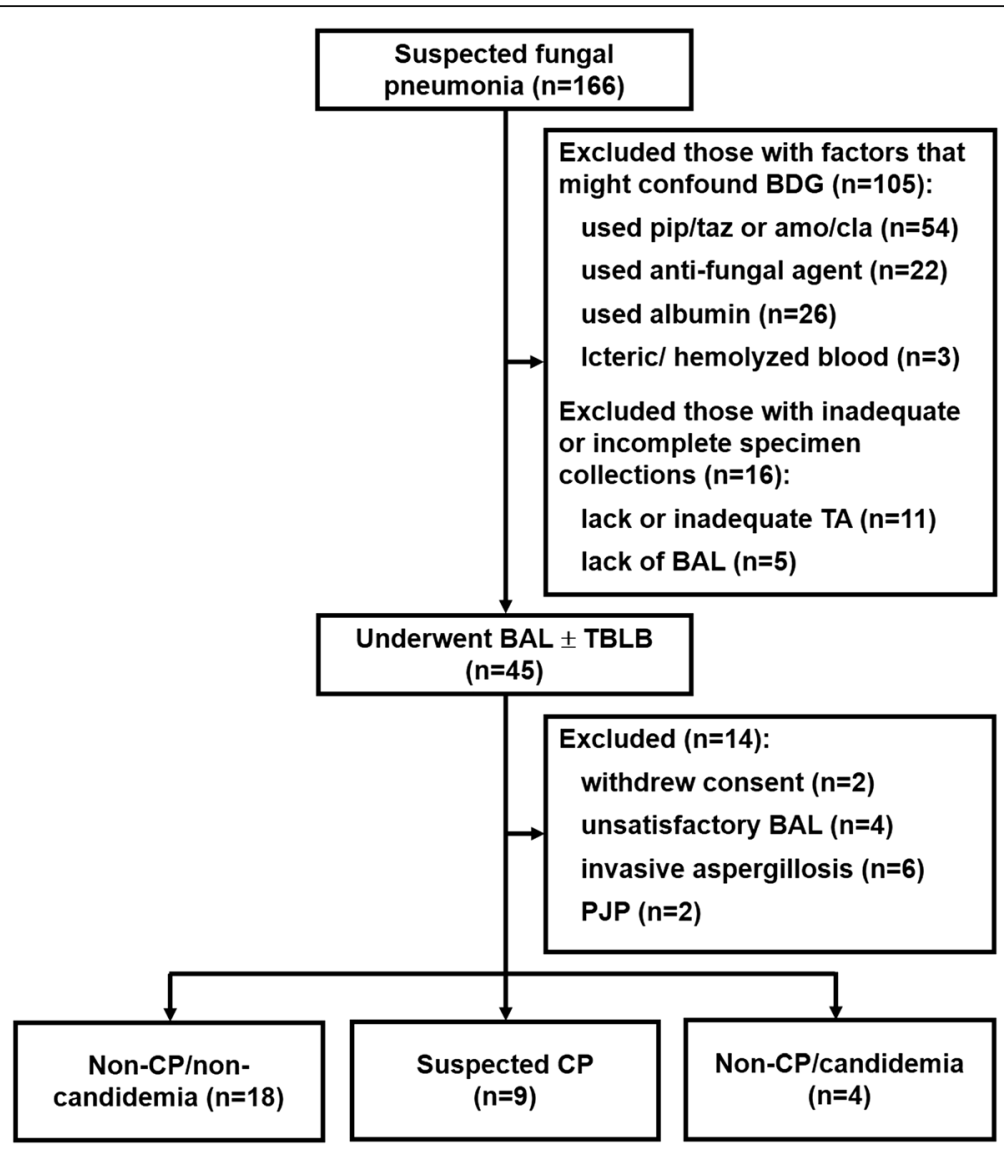

Fig. 1 Study algorithm. pip/taz = piperacillin/tazobactam; amo/cla = amoxicillin-clavulanate; TA = endotracheal aspirate; BAL = bronchoalveolar lavage fluid; TBLB = transbronchial lung biopsy; PJP = Pneumocystis jiroveci pneumonia

\section{Diagnostic definitions}

Suspected CP was defined if the cases met one of the 3 following conditions: 1) histopathological findings of polymorphonuclear leukocytes infiltration in lung, while the presence of hyphae and/or pseudohyphae in the bronchial lumen with or without peribronchial tissue invasion in TBLB specimens; 2) TA and/or BAL cultural yield of Candida spp. with successful antifungal treatment, which was indicated by improvement in attributable symptoms and signs, radiographic resolution of chest images, and clearance of Candida spp. in repeated specimens of TA (at least 2 times per week) or BAL (at the discretion of primary treating team); 3) TA and/or BAL-positive Candida spp. with antifungal treatment failure, which was characterized by persistent growth of Candida spp. in repeated TA or BAL specimens in conjunction with a lack of clinical and radiographic regression. For the later 2 conditions, there were no other reasonably causative factors (such as superinfection of viral or bacterial microorganisms) and the treatment response was modified from the guideline [24] recommended for invasive candidiasis other than CP. Non-CP indicated alternative diagnoses other than suspected CP, which included bacterial pneumonia, viral pneumonitis, pulmonary edema or a combination of these diseases, and all of these cases were discontinued from antifungal treatment within 7 days. Candidemia was defined if the blood culture was positive for Candida spp. growth. Based on these definitions, the final diagnoses were categorized into 3 groups: non-CP/non-candidemia, suspected $\mathrm{CP}$, and non-CP/candidemia.

\section{Management of BDG assay}

All the specimens for the BDG assay were collected and processed within $2 \mathrm{~h}$ of collection, followed by frozen storage at $-70{ }^{\circ} \mathrm{C}$ until testing. The BDG assay was performed (see Additional file 1 for details about methods) using a Glucatell kit (Associates of Cape Cod, Inc., Falmouth, MA, USA) according to the manufacturer's instructions. The results of the BDG assays were not used to categorize the final diagnosis.

\section{Statistical analysis}

Data are presented as the mean $\pm \mathrm{SD}$, the median with inter-quartile range or a number (\%), as appropriate. Continuous variables were evaluated using the KruskalWallis test followed by the Mann-Whitney $U$ test for 
pairwise comparisons. Categorical data were evaluated using the Chi-square or Fisher's exact test. The association between two variables was evaluated using a Pearson's correlation analysis. Analyses of ROC curves were performed to assess the predictive performance of BDG for suspected CP or candidemia in different specimens. The sensitivity, specificity, positive predictive value (PPV), and negative predictive value (NPV) of BDG levels for suspected $\mathrm{CP}$ were also calculated, and the best cutoff values were determined. $P$ values $<0.05$ were considered significant for all tests.

\section{Results}

\section{Patient characteristics}

After enrolling 166 consecutive patients with suspected fungal pneumonia, 31 patients completed the study. The final diagnoses were categorized as non- $\mathrm{CP} /$ non-candidemia $(n=18,58.1 \%)$, suspected CP $(n=9,29.0 \%)$, or non-CP/candidemia ( $n=4,12.9 \%)$ (Fig. 1$)$. In suspected $\mathrm{CP}$ group, there were 4 patients with yeast in TBLB (including one with yeast invasion in peribronchial tissue, Fig. 2) and 5 patients judged by clinical response (3 failure and 2 success). Most of the patients' characteristics were similar among the study groups (Table 1). The ICU mortality rate was $45.2 \%$ in total, but it did not significantly differ among the study group (Table 1). There were no independent factors to predict ICU mortality (please see Additional file 1: Table S1). However, all suspected $\mathrm{CP}$ cases demonstrated yeast evidence based on TBLB histopathology and/or BAL fungal staining, and they also had significantly higher Candida culture rates in BAL (Table 1). Details about the cultural results for the Candida spp. are shown in Additional file 1. All the patients were treated with empirical antifungal agents initially after specimen collection due to concern about immunocompromised status and high severity scores.

\section{BDG levels among the study groups with different diagnoses}

The mean BDG level in TA $(136.6 \pm 37.1 \mathrm{pg} / \mathrm{ml})$ was significant higher in patients with suspected CP than in those in non-CP/non-candidemia $(85.7 \pm 45.1 \mathrm{pg} / \mathrm{ml}$, $P=0.022)$ and non-CP/candidemia $(60.4 \pm 46.6 \mathrm{pg} / \mathrm{ml}$, $P=0.020$ ) (Fig. 3a). The mean BDG level in BAL was also significantly higher in patients with suspected $\mathrm{CP}$ $(217.5 \pm 93.4 \mathrm{pg} / \mathrm{ml})$ than those in the non-CP/non-candidemia $(68.8 \pm 46.5 \mathrm{pg} / \mathrm{ml}, P<0.001)$ and non-CP/candidemia (77.1 $\pm 52.2 \mathrm{pg} / \mathrm{ml}, P=0.003$ ) groups (Fig. $3 \mathrm{~b}$ ). Conversely, the mean BDG level in serum in the non$\mathrm{CP} /$ candidemia group $(221.1 \pm 96.0 \mathrm{pg} / \mathrm{ml})$ was significantly higher than that in the non- $\mathrm{CP} /$ non-candidemia group $(20.1 \pm 7.5 \mathrm{pg} / \mathrm{ml}, P<0.001)$ or the suspected $\mathrm{CP}$ group $(22.7 \pm 4.5 \mathrm{pg} / \mathrm{ml}, P<0.001)$ (Fig. $3 \mathrm{c}$ ).

\section{BDG level and Candida culture results}

In all the patients, the BDG level was significantly higher in specimens with growth of Candida spp. than in those with a negative yield in each of the 3 types of specimens $(126.2 \pm 34.8$ vs. $73.3 \pm 47.9 \mathrm{pg} / \mathrm{ml}, P=0.004$ in $\mathrm{TA}$; $191.0 \pm 92.1$ vs. $56.7 \pm 30.2 \mathrm{pg} / \mathrm{ml}, P<0.001$ in BAL;

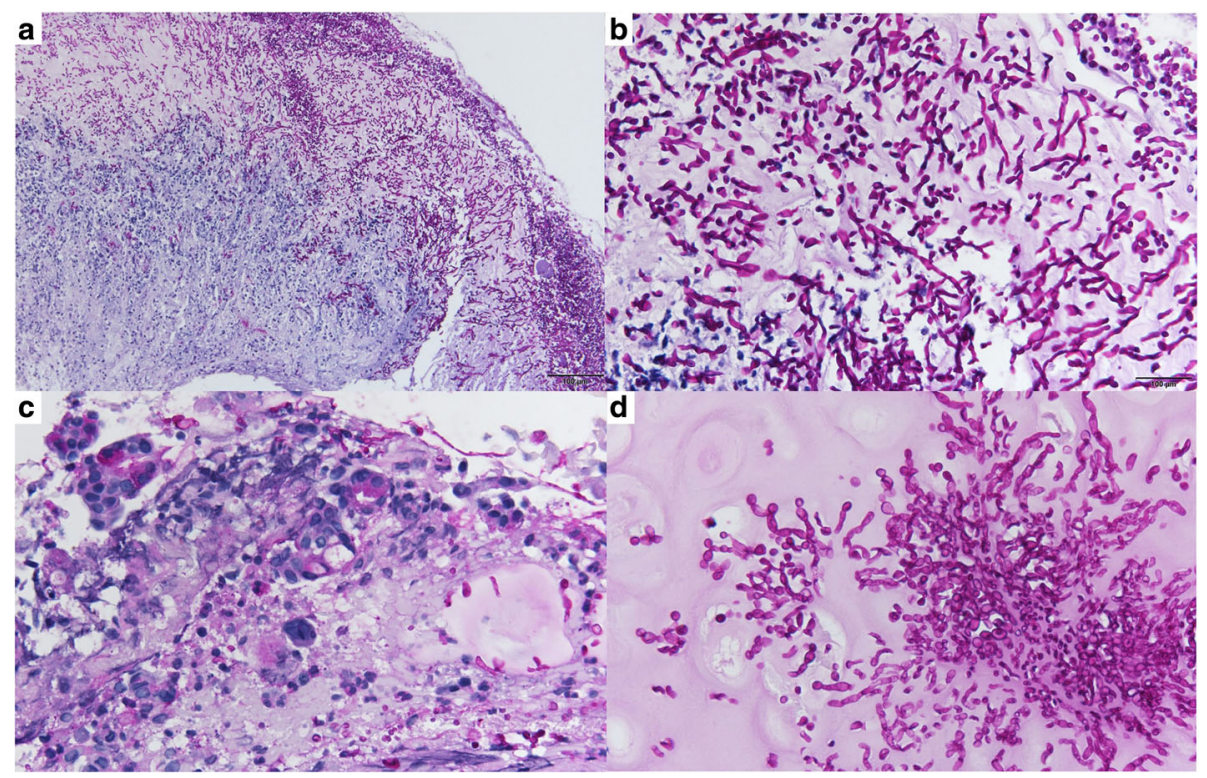

Fig. 2 Histological findings of transbronchial lung biopsy with Periodic Acid-Schiff stain. A representative section of necrotic tissue admixed with inflammatory exudate showed infiltrations of acute inflammatory cells and fungus colonies in low magnification (a, 100X) as well as budding yeast and hyphae/pseudohyphae appearance in high magnification (b, 400X). Sections of peribronchial tissue disclosed epithelial sloughing with yeast invasion in bronchial mucosa (c, 400X) and bronchial cartilage (d, 400X) 
Table 1 Patients' characteristics

\begin{tabular}{|c|c|c|c|c|c|}
\hline & All & Non-CP/non- candidemia & Suspected CP & Non-CP/ candidemia & $P$ \\
\hline Number & 31 & 18 & 9 & 4 & \\
\hline Age, year & $66.4 \pm 16.6$ & $65.3 \pm 18.5$ & $66.9 \pm 16.5$ & $70.3 \pm 6.2$ & $0.971^{\mathrm{a}}$ \\
\hline Sex, male (\%) & $20(64.5)$ & $11(61.1)$ & $6(66.7)$ & $3(75.0)$ & $0.860^{b}$ \\
\hline APACHE II at ICU admission & $22.4 \pm 5.2$ & $22.3 \pm 5.2$ & $21.1 \pm 4.2$ & $25.3 \pm 6.9$ & $0.583^{\mathrm{a}}$ \\
\hline APACHE II on enrolled day & $24.2 \pm 4.8$ & $23.2 \pm 5.0$ & $25.1 \pm 3.9$ & $27.0 \pm 5.3$ & $0.316^{\mathrm{a}}$ \\
\hline \multicolumn{6}{|l|}{ Initial reasons for MV (\%) } \\
\hline Post-surgery & $7(22.6 \%)$ & $3(16.7)$ & $2(22.2)$ & $2(50.0)$ & $0.353^{b}$ \\
\hline Pneumonia & $24(77.4 \%)$ & $15(83.3)$ & $7(77.8)$ & $2(50.0)$ & $0.353^{b}$ \\
\hline \multicolumn{6}{|l|}{ Reasons for BAL (\%) } \\
\hline Non-responding pneumonia & $18(58.1)$ & $9(50.0)$ & $7(77.8)$ & $2(50)$ & $0.363^{b}$ \\
\hline Newly developed infiltration & $13(41.9)$ & $9(50.0)$ & $2(22.2)$ & $2(50)$ & $0.363^{b}$ \\
\hline \multicolumn{6}{|l|}{ Host factors (\%) } \\
\hline Recent neutropenia & $10(32.3)$ & $5(27.8)$ & $2(22.2)$ & $3(75.0)$ & $0.141^{b}$ \\
\hline Prolonged steroid use & $16(51.6)$ & $10(55.6)$ & $5(55.6)$ & $1(25.0)$ & $0.521^{b}$ \\
\hline Immunosuppressants & $5(16.1)$ & $3(16.7)$ & $2(22.2)$ & 0 & $0.600^{b}$ \\
\hline MV days & $24.2 \pm 12.5$ & $23.7 \pm 14.9$ & $22.9 \pm 9.4$ & $29.3 \pm 5.7$ & $0.129^{a}$ \\
\hline ICU stay, days & $27.8 \pm 11.9$ & $28.0 \pm 14.4$ & $26.6 \pm 9.2$ & $30.0 \pm 4.3$ & $0.494^{\mathrm{a}}$ \\
\hline ICU mortality (\%) & $14(45.2)$ & $6(33.3)$ & $5(55.6)$ & $3(75.0)$ & $0.241^{b}$ \\
\hline \multicolumn{6}{|l|}{ Radiographic manifestations ${ }^{\mathrm{C}}(\%)$} \\
\hline Halo sign & $1(3.2)$ & 0 & $1(11.1)$ & 0 & $0.283^{b}$ \\
\hline Single/multiple nodules & $11(35.5)$ & $3(16.7)$ & $7(77.8)$ & $1(25.0)$ & $0.007^{b}$ \\
\hline Cavitary lesion & $6(19.4)$ & $3(16.7)$ & $3(33.3)$ & 0 & $0.338^{b}$ \\
\hline GGO/infiltration & $29(93.5)$ & $17(94.4)$ & $8(88.9)$ & $4(100)$ & $0.732^{b}$ \\
\hline \multicolumn{6}{|l|}{ Mycological evidence of yeast (\%) } \\
\hline $\mathrm{TBLB}^{\mathrm{d}, \mathrm{e}}$ & $4(12.9)$ & 0 & $4(44.5)$ & 0 & $<0.001^{b}$ \\
\hline $\mathrm{BAL}^{\mathrm{e}}$ & $13(41.9)$ & $4(22.2)$ & $8(88.9)$ & $1(25.0)$ & $0.003^{b}$ \\
\hline TA fungal culture & $14(45.2)$ & $7(38.9)$ & $6(66.7)$ & $1(25.0)$ & $0.269^{b}$ \\
\hline BAL fungal culture & $13(41.9)$ & $5(27.8)$ & $8(88.9)$ & 0 & $0.002^{b}$ \\
\hline
\end{tabular}

Data are presented with means \pm standard deviations

$C P$ Candida pneumonia, $A P A C H E$ acute physiology and chronic health evaluation, $M V$ mechanical ventilation, $B A L$ bronchoalveolar lavage, GGO ground glass opacity, $T B L B$ transbronchial lung biopsy, $T A$ endotracheal aspirate, $B A L$ bronchoalveolar lavage fluid

${ }^{a}$ Kruskal-Wallis test

${ }^{b}$ Fisher's exact test

${ }^{\mathrm{C}}$ Each single patient could have more than one type of radiographic manifestation

${ }^{\mathrm{d}} \mathrm{A}$ total of 19 patients underwent TBLB, including 11 in non-CP/non-candidemia, 7 in suspected CP, and 1 in non-CP/candidemia

${ }^{e}$ Fungal staining was applied using Grocott-Gomori methenamine silver stain in BAL and Periodic Acid-Schiff stain in TBLB

$221.1 \pm 96.0$ vs. $21.0 \pm 6.7 \mathrm{pg} / \mathrm{ml}, P=0.001$ in serum) (Fig. 4a). Additionally, the value of TA BDG was positively correlated with that of BAL BDG $(P=0.002, r=0.542)$ (Fig. 4b). However, the value of neither TA BDG $(P=0.598 ; r=0.098)$ nor BAL BDG $(P=0.836 ; r=0.039)$ was significantly correlated with that of serum BDG.

\section{Diagnostic value of BDG in detecting suspected $\mathrm{CP}$}

For the detection of suspected CP, measuring BDG levels in both TA and BAL, but not the serum BDG level, could have good diagnostic value. The results from the ROC analysis revealed that BDG levels in TA and BAL had
AUROC values of 0.833 and 0.939 , respectively (Fig. 5). Given a cutoff BDG level of $120 \mathrm{pg} / \mathrm{ml}$ in TA and $130 \mathrm{pg} /$ $\mathrm{ml}$ in BAL, the best diagnostic value could be achieved with sensitivity/specificity of $67 \% / 82 \%$ for TA and $89 \% /$ $86 \%$ for BAL (Table 2). The serum BDG level exerted excellent predictive performance for the detection of candidemia (AUROC $=0.996, P=0.001$ ) but poor predictive performance for the detection of suspected CP (AUROC $=0.510, P=0.931$, Fig. 5). In addition, measuring the BDG level in TA (AUROC $=0.259, P=0.126$ ) or BAL (AUROC $=0.389, P=0.480)$ for the detection of candidemia was of no diagnostic value. 


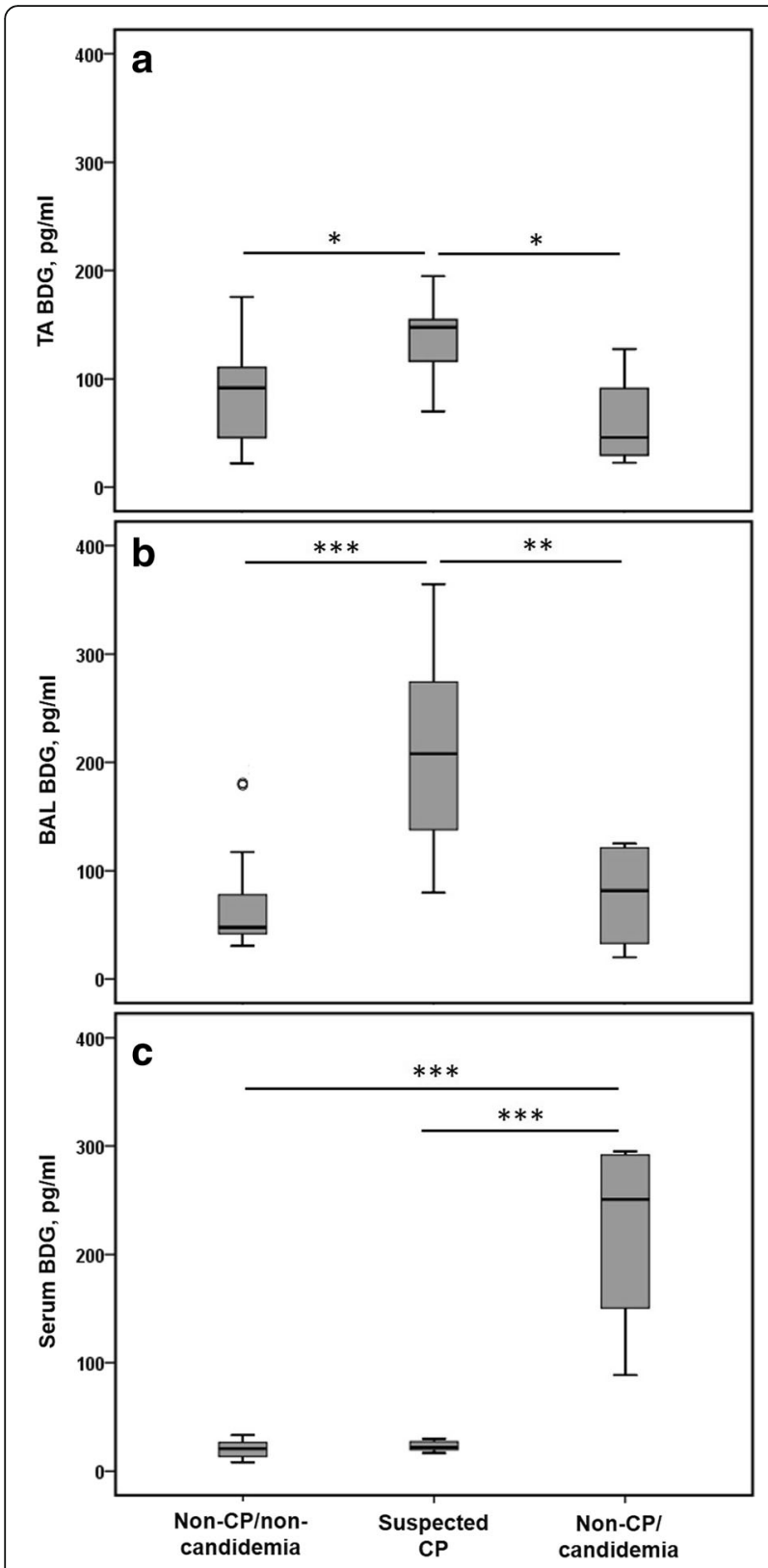

Fig. 3 The levels of (1,3)-B-D-glucan (BDG) in specimens of endotracheal aspirate (a, TA), bronchoalveolar lavage fluid (b, BAL), and serum (c), categorized by different diagnoses. $\mathrm{CP}=$ Candida pneumonia. The shallow cycle indicates overlap of 2 outliers (180.8 and $178.0 \mathrm{pg} / \mathrm{ml}$ ). ${ }^{*} P<0.05$; ${ }^{*} P<0.01,{ }^{* * *} P<0.001$. Statistical evaluations were performed using the Kruskal-Wallis test followed by the Mann-Whitney $U$ test

\section{Discussion}

To our knowledge, the present study was the first to compare the diagnostic value of measuring BDG levels in TA, $\mathrm{BAL}$, and serum for the detection of suspected CP in immunocompromised and critically ill patients. The major findings were that measuring BDG levels in respiratory specimens, including both TA and BAL fluid, exerted good diagnostic value for the detection of suspected $\mathrm{CP}$, particularly in the absence of concurrent candidemia. In contrast, measuring the serum BDG level had no diagnostic value for this detection.

There are limited data regarding measuring BAL BDG levels to diagnose fungal pneumonia. The performance of BAL BDG at different cutoff value to diagnose non-Candida fungal pneumonia varied widely, in terms of sensitivity (53-90\%) and specificity (26-88\%) in different patient populations [25-28]. Among these, the largest study (268 BALs) reported by Prattes et al., who compared BAL galactomannan and BDG to diagnose proven/probable invasive pulmonary aspergillosis. They concluded that despite similar sensitivity (> 90\%) and NPV (> 90\%) between galactomannan and BDG, BAL BDG was less convincing due to low specificity $(<50 \%)$. This might be ascribed to frequent respiratory colonization of Candida spp. [28], which potentially limited the diagnostic performance of BAL BDG for non-Candida fungal pneumonia. In contrast, we excluded most of the possible confounding factors and focused on Candida pathogens. Thus, our data showed good performance (both sensitivity and specificity $>85 \%$ ) by means of measuring BAL BDG. Moreover, consistent with the observation reported by Reischies et al. [29], our data also showed that Candida culture-positive specimens had higher BDG levels (Fig. 4a), which implicates that BDG levels reflect fungal burdens indirectly. Patients with higher fungal burdens are more susceptible to fungal infection. This potentially provide the rationale to measure respiratory BDG levels as good diagnostic aid to detect suspected CP.

We further showed that the TA BDG level was positively correlated with BAL BDG (Fig. 4b). Despite with inferior value to BAL BDG, measuring TA BDG also offered good diagnostic performance for the detection of suspected $\mathrm{CP}$ (Fig. 5). Thus, measuring TA BDG could serve as a simple, less-invasive, alternative diagnostic for suspected CP when BAL is unavailable. Previously, most cases of CP were considered to indicate lung involvement of disseminated candidiasis rather than primary $\mathrm{CP}[1,5]$. Based on this belief, patients with $\mathrm{CP}$ are supposed to have as high of a serum BDG level as those with candidemia. However, our data did not correspond to this notion. Patients with suspected CP had apparently low BDG in serum (vs. those with non-CP/candidemia) and high BDG in TA and BAL (Fig. 3). These findings suggested that serum BDG might have no diagnostic value in patients with suspected $C P$ in the absence of concurrent candidemia. Thus, we suggest clinicians should directly measure BDG in respiratory specimens in patients with suspicion of CP.

To date, there have been no universally acceptable criteria to diagnose $\mathrm{CP}$, and most diagnoses have depended on lung biopsies. CP is considered uncommon based on earlier lung autopsy reports $[1,6,8]$, so the clinical practice guideline does not recommend using antifungal treatment based upon the Candida culture alone $[3,5,30]$. 

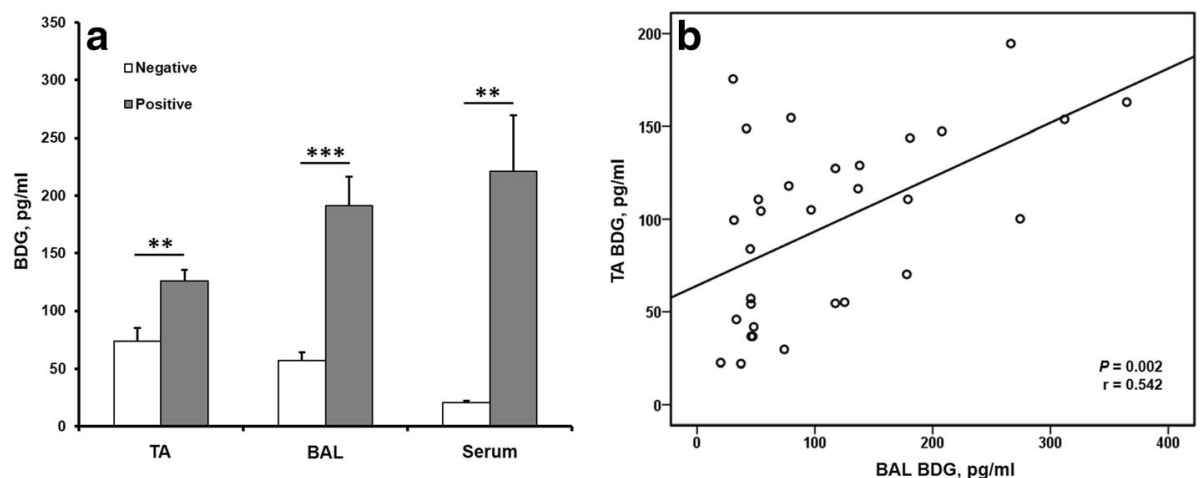

Fig. 4 a BDG levels categorized by fungal cultural results in different types of specimens. ${ }^{*} P<0.01,{ }^{* *} P<0.001$. TA $=$ endotracheal aspirate; $\mathrm{BAL}=$ bronchoalveolar lavage fluid. $\mathbf{b}$ Binary correlation of values of TA BDG and BAL BDG. $r=$ correlation coefficient. Statistical evaluations were performed using the Mann-Whitney $U$ test in A and using Pearson's correlation coefficient in $\mathbf{b}$

However, CP might possibly be under-diagnosed in critically ill patients because a lung biopsy is rarely performed. Indeed, even for immunocompetent ICU patients with Candida-positive respiratory specimens, $24.2 \%$ of physicians recommended antifungal treatment in an early questionnaire surveillance regarding $\mathrm{CP}$ [31] and $32.9 \%$ did so in a recent prospective study concerning ICU-acquired pneumonia [32]. These reports reflected the dissociation of managements between the guideline $[3,5,30]$ and reallife ICU situations remain an unresolved issue. It is logically speculated that antifungal treatment are more frequently used for immunocompromised and critically ill ICU patients, particularly while these patients were undergoing non-responding or progressive pneumonia with the

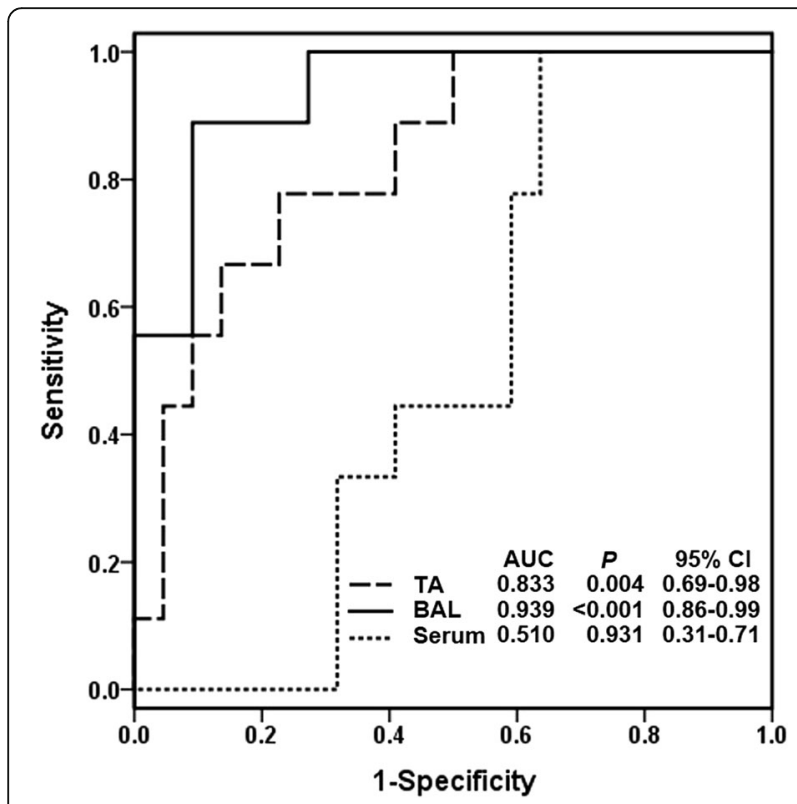

Fig. 5 The receiver operating characteristic curve of BDG levels in different types of specimens for the diagnosis of suspected Candida pneumonia (CP). TA = endotracheal aspirate; $\mathrm{BAL}=$ bronchoalveolar lavage fluid; $\mathrm{AUC}=$ area under the curve; $\mathrm{Cl}=$ conference interval presence of respiratory Candida spp. and the absence of the other causative pathogens after thorough work-ups. Thus, applying antifungal treatment response in these situations might be reasonably therapeutic diagnostics for suspected $\mathrm{CP}$ and could compensate the gap between the

Table 2 Predictive performance of various BDG levels for diagnosing candidemia and suspected CP in different specimens

\begin{tabular}{lllll}
\hline Cutoff value & Sensitivity & Specificity & PPV & NPV \\
\hline Candidemia & & & & \\
Serum BDG, pg/ml & & & & \\
70 & $100 \%$ & $100 \%$ & $100 \%$ & $100 \%$ \\
80 & $100 \%$ & $100 \%$ & $100 \%$ & $100 \%$ \\
90 & $75 \%$ & $100 \%$ & $100 \%$ & $96 \%$
\end{tabular}

Suspected CP

$\mathrm{TA} B \mathrm{BDG}, \mathrm{pg} / \mathrm{ml}^{\mathrm{a}}$

$\begin{array}{lllll}90 & 89 \% & 55 \% & 44 \% & 92 \% \\ 100 & 89 \% & 59 \% & 47 \% & 93 \% \\ 110 & 78 \% & 68 \% & 50 \% & 88 \% \\ 120 & 67 \% & 82 \% & 60 \% & 86 \% \\ 130 & 0 \% & 70 \% & 0 \% & 83 \%\end{array}$

BAL BDG, pg/ml

$\begin{array}{lllll}110 & 89 \% & 73 \% & 57 \% & 94 \% \\ 120 & 89 \% & 77 \% & 62 \% & 94 \% \\ 130 & 89 \% & 86 \% & 73 \% & 95 \% \\ 140 & 89 \% & 91 \% & 80 \% & 95 \% \\ 150 & 67 \% & 91 \% & 75 \% & 95 \%\end{array}$

Serum BDG, pg/ml

\begin{tabular}{lllll}
20 & $67 \%$ & $41 \%$ & $32 \%$ & $75 \%$ \\
25 & $33 \%$ & $59 \%$ & $25 \%$ & $68 \%$ \\
30 & $0 \%$ & $73 \%$ & $0 \%$ & $64 \%$ \\
\hline
\end{tabular}

$P P V$ positive predictive value, $N P V$ negative predictive value, $B D G \beta$-D-glucan Refer to Table 1 for other abbreviations

${ }^{\mathrm{a}}$ Adjusted to per gram of endotracheal aspirate 
guideline and clinical scenario. Accordingly, more practical diagnostic criteria for detecting suspected CP are urgently needed. Our findings showed measuring respiratory BDG levels might provide valuable information to enhance these diagnostic criteria.

There were some limitations of our study. First, our data were derived from selected patients to minimize confounding factors. Particularly, we excluded many patients with concurrent use of antibiotics. The concern that if concurrent use of antibiotics possibly confounded BDG assay remains a conflicting issue [33-40]. It needs a large-scale study to validate this issue. Second, the sample size of this study was small, and future investigations with larger sample sizes will be needed to optimize the best TA or BAL BDG cutoff values. Third, our results were derived from the study of Candida spp.; the application of measuring BDG levels in other fungal pathogens requires further validation.

\section{Conclusions}

This study is not intended to replace lung biopsy for the diagnosis of $\mathrm{CP}$, but try to use less invasive biomarker to detect suspected CP. Our results clearly showed that the BDG level in BAL or TA provided a good diagnostic value to detect suspected CP. Additionally, measuring serum BDG had no diagnostic value for detecting suspected $\mathrm{CP}$ in the absence of concurrent candidemia. These findings warrant a future large-scale investigation to validate the optimal cutoff value for BDG in respiratory specimens and to determine the timing to initiate antifungal treatment.

\section{Additional file}

Additional file 1: Supplementary Materials. (DOCX 24 kb)

\section{Abbreviations \\ AUROC: Area under the receiver operating characteristic curve; \\ BAL: Bronchoalveolar lavage; BDG: (1,3)- $\beta$-D-glucan; CP: Candida pneumonia; ICU: Intensive care unit; NPV: Negative predictive value; PPV: Positive predictive value; TA: Endotracheal aspirate; TBLB: Transbronchial lung biopsy}

\section{Acknowledgements}

We appreciated the great assistance of Dr. Yi-Chen, from Department of Pathology and Laboratory Medicine in Taipei Veterans General Hospital, for the preparation of histopathological findings of lung biopsy. We thank all the study participants and the Division of Experimental Surgery of the Department of Surgery for their assistance in part of laboratory work.

\section{Funding}

This study was supported by a grant V100B-032 from Taipei Veterans General Hospital, Taipei, Taiwan, and in part by grants MOST 104-2320-B-010-014MY3, NSC 98-2628-B-320-003-MY3, and MOST 105-2314-B-075-034 from the Ministry of Science and Technology, Taiwan.

\section{Availability of data and materials}

All data generated during this study are included in this published article and its supplementary information files.

\section{Authors' contributions}

YRK and DWP had full access to all the data in the study and takes responsibility for the integrity of the data and the accuracy of the data analysis. KCS, DWP, YRK, and YCL contributed to concept generation and study design. $\mathrm{KTC}, \mathrm{YHH}, \mathrm{CMT}$ and VYS contributed to data acquisition, analysis, and the first draft. KCS contributed to grant application, data interpretation, and manuscript revision. YRK and DWP revised the manuscript critically for important

intellectual content. All authors read and approved the final manuscript.

\section{Competing interests}

The authors declare that they have no competing interests.

\section{Consent for publication}

Not applicable.

\section{Ethics approval and consent to participate}

This study was approved by the institutional review board of Taipei Veterans General Hospital (IRB approval ID: 201011002IA) and signed informed consents were obtained from all participants.

\section{Publisher's note}

Springer Nature remains neutral with regard to jurisdictional claims in published maps and institutional affiliations.

\section{Author details}

'Department of Chest Medicine, Taipei Veterans General Hospital, No.201, Sec. 2, Shipai Rd., Beitou Dist., Taipei City 11217, Taiwan, Republic of China. ${ }^{2}$ Center of Sleep Medicine, Taipei Veterans General Hospital, No.201, Sec. 2, Shipai Rd., Beitou Dist., Taipei City 11217, Taiwan, Republic of China. ${ }^{3}$ Institute of Physiology, School of Medicine, National Yang-Ming University, No.155, Sec.2, Linong St., Beitou Dist., Taipei City 11221, Taiwan, Republic of China. ${ }^{4}$ Division of Thoracic Medicine, Department of Medicine, Cheng Hsin General Hospital, No.45, Cheng Hsin St., Beitou Dist., Taipei City 11220, Taiwan, Republic of China. ${ }^{5}$ Sijhih Cathay General Hospital, No.2, Ln. 59, Jiancheng Rd., Xizhi Dist., New Taipei City 22174, Taiwan, Republic of China. ${ }^{6}$ School of Medicine, National Yang-Ming University, No.155, Sec.2, Linong St., Beitou Dist., Taipei City 11221, Taiwan, Republic of China.

Received: 26 December 2016 Accepted: 29 March 2017 Published online: 08 April 2017

\section{References}

1. Haron E, Vartivarian S, Anaissie E, Dekmezian R, Bodey GP. Primary Candida pneumonia. Experience at a large cancer center and review of the literature. Medicine (Baltimore). 1993;72:137-42.

2. Ascioglu S, Rex JH, de Pauw B, Bennett JE, Bille J, Crokaert F, et al. Defining opportunistic invasive fungal infections in immunocompromised patients with cancer and hematopoietic stem cell transplants: an international consensus. Clin Infect Dis. 2002;34:7-14.

3. De Pauw B, Walsh TJ, Donnelly JP, Stevens DA, Edwards JE, Calandra T, et al. Revised definitions of invasive fungal disease from the European Organization for Research and Treatment of cancer/invasive fungal infections cooperative group and the National Institute of Allergy and Infectious Diseases mycoses study group (EORTC/MSG) consensus group. Clin Infect Dis. 2008;46:1813-21.

4. Levi M, Opal SM. Coagulation abnormalities in critically ill patients. Crit Care. 2006;10:222

5. Pappas PG, Kauffman CA, Andes D, Benjamin Jr DK, Calandra TF, Edwards Jr JE, et al. Clinical practice guidelines for the management of candidiasis: 2009 update by the Infectious Diseases Society of America. Clin Infect Dis. 2009;48:503-35.

6. Kontoyiannis DP, Reddy BT, Torres HA, Luna M, Lewis RE, Tarrand J, et al. Pulmonary candidiasis in patients with cancer: an autopsy study. Clin Infect Dis. 2002:34:400-3.

7. Rello J, Esandi ME, Diaz E, Mariscal D, Gallego M, Valles J. The role of Candida sp isolated from bronchoscopic samples in nonneutropenic patients. Chest. 1998;114:146-9.

8. el-Ebiary M, Torres A, Fabregas N, de la Bellacasa JP, Gonzalez J, Ramirez J, et al. Significance of the isolation of Candida species from respiratory samples in critically ill, non-neutropenic patients. An immediate postmortem histologic study. Am J Respir Crit Care Med. 1997;156:583-90. 
9. Wood GC, Mueller EW, Croce MA, Boucher BA, Fabian TC. Candida sp. isolated from bronchoalveolar lavage: clinical significance in critically ill trauma patients. Intensive Care Med. 2006;32:599-603.

10. Ostrosky-Zeichner L. Invasive mycoses: diagnostic challenges. Am J Med. 2012;125:S14-24.

11. Albert M, Williamson D, Muscedere J, Lauzier F, Rotstein C, Kanji S, et al, Candida in the respiratory tract secretions of critically ill patients and the impact of antifungal treatment: a randomized placebo controlled pilot trial (CANTREAT study). Intensive Care Med. 2014;40:1313-22.

12. Ricard JD, Roux D. Candida colonization in ventilated ICU patients: no longer a bystander! Intensive Care Med. 2012;38:1243-5.

13. Ricard JD, Roux D. Candida pneumonia in the ICU: myth or reality? Intensive Care Med. 2009;35:1500-2.

14. Azoulay E, Timsit JF, Tafflet M, de Lassence A, Darmon M, Zahar JR, et al. Candida colonization of the respiratory tract and subsequent pseudomonas ventilator-associated pneumonia. Chest. 2006;129:110-7.

15. Nseir S, Jozefowicz E, Cavestri B, Sendid B, Di Pompeo C, Dewavrin F, et al. Impact of antifungal treatment on Candida-pseudomonas interaction: a preliminary retrospective case-control study. Intensive Care Med. 2007;33:137-42.

16. Tan X, Zhu S, Yan D, Chen W, Chen R, Zou J, et al. Candida spp. airway colonization: a potential risk factor for Acinetobacter baumannii ventilatorassociated pneumonia. Med Mycol. 2016;54:557-66.

17. Hamet M, Pavon A, Dalle F, Pechinot A, Prin S, Quenot JP, et al. Candida spp. airway colonization could promote antibiotic-resistant bacteria selection in patients with suspected ventilator-associated pneumonia. Intensive Care Med. 2012;38:1272-9.

18. Delisle MS, Williamson DR, Perreault MM, Albert M, Jiang X, Heyland DK. The clinical significance of Candida colonization of respiratory tract secretions in critically ill patients. J Crit Care. 2008;23:11-7.

19. Delisle MS, Williamson DR, Albert M, Perreault MM, Jiang X, Day AG, et al. Impact of Candida species on clinical outcomes in patients with suspected ventilator-associated pneumonia. Can Respir J. 2011;18:131-6.

20. Karageorgopoulos DE, Vouloumanou EK, Ntziora F, Michalopoulos A, Rafailidis PI, Falagas ME. beta-D-glucan assay for the diagnosis of invasive fungal infections: a meta-analysis. Clin Infect Dis. 2011;52:750-70.

21. Hsu JL, Ruoss SJ, Bower ND, Lin M, Holodniy M, Stevens DA. Diagnosing invasive fungal disease in critically ill patients. Crit Rev Microbiol. 2011;37:277-312.

22. Alexander BD, Smith PB, Davis RD, Perfect JR, Reller LB. The $(1,3)\{$ beta\}-Dglucan test as an aid to early diagnosis of invasive fungal infections following lung transplantation. J Clin Microbiol. 2010;48:4083-8.

23. Pickering JW, Sant HW, Bowles CA, Roberts WL, Woods GL. Evaluation of a (1->3)-beta-D-glucan assay for diagnosis of invasive fungal infections. J Clin Microbiol. 2005;43:5957-62.

24. Segal BH, Herbrecht R, Stevens DA, Ostrosky-Zeichner L, Sobel J, Viscoli C, et al. Defining responses to therapy and study outcomes in clinical trials of invasive fungal diseases: Mycoses Study Group and European Organization for Research and Treatment of Cancer consensus criteria. Clin Infect Dis. 2008:47:674-83.

25. Mutschlechner W, Risslegger B, Willinger B, Hoenigl M, Bucher B, Eschertzhuber S, et al. Bronchoalveolar Lavage fluid (1,3)beta-D-Glucan for the diagnosis of invasive fungal infections in solid organ transplantation: a prospective multicenter study. Transplantation. 2015;99:e140-4.

26. Rose SR, Vallabhajosyula S, Velez MG, Fedorko DP, VanRaden MJ, Gea-Banacloche

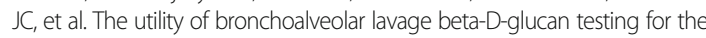
diagnosis of invasive fungal infections. J Inf Secur. 2014;69:278-83.

27. Hoenigl M, Prattes J, Spiess B, Wagner J, Prueller F, Raggam RB, et al. Performance of galactomannan, beta-d-glucan, Aspergillus lateral-flow device, conventional culture, and PCR tests with bronchoalveolar lavage fluid for diagnosis of invasive pulmonary aspergillosis. J Clin Microbiol. 2014;52:2039-45.

28. Prattes J, Flick H, Pruller F, Koidl C, Raggam RB, Palfner M, et al. Novel tests for diagnosis of invasive aspergillosis in patients with underlying respiratory diseases. Am J Respir Crit Care Med. 2014;190:922-9.

29. Reischies FM, Prattes J, Pruller F, Eigl S, List A, Wolfler A, et al. Prognostic potential of 1,3-beta-d-glucan levels in bronchoalveolar lavage fluid samples. J Inf Secur. 2016;72:29-35.

30. Pappas PG, Kauffman CA, Andes DR, Clancy CJ, Marr KA, Ostrosky-Zeichner L et al. Clinical practice guideline for the Management of Candidiasis: 2016 update by the Infectious Diseases Society of America. Clin Infect Dis. 2016;62:e1-e50.

31. Azoulay E, Cohen Y, Zahar JR, Garrouste-Orgeas M, Adrie C, Moine P, et al. Practices in non-neutropenic ICU patients with Candida-positive airway specimens. Intensive Care Med. 2004;30:1384-9.
32. Terraneo S, Ferrer M, Martin-Loeches I, Esperatti M, Di Pasquale M, Giunta V, et al. Impact of Candida spp. isolation in the respiratory tract in patients with intensive care unit-acquired pneumonia. Clin Microbiol Infect. 2016; 22(94):e1-8.

33. Liss B, Cornely OA, Hoffmann D, Dimitriou V, Wisplinghoff H. 1,3-beta-dGlucan contamination of common antimicrobials. J Antimicrob Chemoth. 2016;71:913-5.

34. Liss B, Cornely OA, Hoffmann D, Dimitriou V, Wisplinghoff H. 1,3-beta-DGlucan contamination of common antimicrobials-authors' response. J Antimicrob Chemoth. 2016; 71:2997-2999.

35. Finkelman MA. Comment on: 1,3-beta-D-Glucan contamination of common antimicrobials. J Antimicrob Chemoth. 2016;71:2996-7.

36. Hammarstrom $\mathrm{H}$, Kondori $\mathrm{N}$, Friman $\mathrm{V}$, Wenneras $\mathrm{C}$. How to interpret serum levels of beta-glucan for the diagnosis of invasive fungal infections in adult high-risk hematology patients: optimal cut-off levels and confounding factors. Eur J Clin Microbiol. 2015;34:917-25.

37. Metan G, Agkus C, Koc AN, Elmali F, Finkelman MA. Does ampicillinsulbactam cause false positivity of (1,3)-beta-D-glucan assay? A prospective evaluation of 15 patients without invasive fungal infections. Mycoses. 2012; 55:366-71.

38. Mennink-Kersten MA, Warris A, Verweij PE. 1,3-beta-D-glucan in patients receiving intravenous amoxicillin-clavulanic acid. N Engl J Med. 2006;354: 2834-5.

39. Metan G, Agkus C, Buldu H, Koc AN. The interaction between piperacillin/ tazobactam and assays for Aspergillus galactomannan and 1,3-beta-Dglucan in patients without risk factors for invasive fungal infections. Infection. 2010;38:217-21.

40. Marty FM, Lowry CM, Lempitski SJ, Kubiak DW, Finkelman MA, Baden LR. Reactivity of (1->3)-beta-d-glucan assay with commonly used intravenous antimicrobials. Antimicrob Agents Chemother. 2006;50:3450-3.

\section{Submit your next manuscript to BioMed Central and we will help you at every step:}

- We accept pre-submission inquiries

- Our selector tool helps you to find the most relevant journal

- We provide round the clock customer support

- Convenient online submission

- Thorough peer review

- Inclusion in PubMed and all major indexing services

- Maximum visibility for your research

Submit your manuscript at www.biomedcentral.com/submit
) Biomed Central 